\title{
Effects of Violating Detailed Balance on Critical Dynamics
}

\author{
Uwe C. Täuber, ${ }^{1}$ Vamsi K. Akkineni, ${ }^{1,2}$ and Jaimie E. Santos ${ }^{3}$ \\ ${ }^{1}$ Department of Physics, Virginia Tech, Blacksburg, Virginia 24061-0435 \\ ${ }^{2}$ Department of Physics, University of Illinois at Urbana-Champaign, Urbana, Illinois 61801-3080 \\ ${ }^{3}$ Hahn-Meitner-Institut, Abteilung Theorie, D-14109 Berlin, Germany
}

(Received 25 September 2001; published 14 January 2002)

\begin{abstract}
We present an overview of the effects of detailed-balance violating perturbations on the universal static and dynamic scaling behavior near a critical point. It is demonstrated that the standard critical dynamics universality classes are generally quite robust: In systems with nonconserved order parameter, detailed balance is effectively restored at criticality. This also holds for models with conserved order parameter, and isotropic nonequilibrium perturbations. Genuinely novel features are found only for models with conserved order parameter and spatially anisotropic noise correlations.
\end{abstract}

DOI: 10.1103/PhysRevLett.88.045702

One of the major goals in theoretical nonequilibrium physics has been the identification and classification of universality classes for the long-wavelength, long-time scaling behavior both near continuous dynamical phase transitions, and for systems displaying generic scale invariance. Indeed, through investigations of certain specific models, a number of prototypical nonequilibrium universality classes have been identified. Prominent examples are driven diffusive systems (DDS) [1], models of driven interfaces and growing surfaces [2], depinning transitions [3], and phase transitions from active to absorbing states [4], e.g., in diffusion-limited chemical reactions.

A complementary approach is to study the influence of nonequilibrium perturbations on the known universality classes for equilibrium dynamical critical phenomena [5]. Equilibrium critical dynamics is concerned with the relaxational and reversible kinetics near a thermodynamic critical point at temperature $T_{c}$, as generically described by the Landau-Ginzburg-Wilson (LGW) model for an $n$ component order parameter vector field $\mathbf{S}$ in $d$ space dimensions [6]. In addition to the two independent static critical exponents, e.g., the correlation length exponent $\nu$ defined via $\xi \propto|\tau|^{-\nu}\left(\tau=T-T_{c}\right)$ and Fisher's exponent $\eta$ for the algebraic decay of the two-point correlation function at criticality $\left(T=T_{c}\right), C\left(x-x^{\prime}\right) \propto \mid x-$ $\left.x^{\prime}\right|^{-(d-2+\eta)}$, the order parameter relaxation is governed by a dynamic exponent $z$ that describes critical slowing down: The characteristic time scale diverges as $t_{\mathrm{ch}} \propto$ $|\tau|^{-z \nu}$ upon approaching the transition. This allows for time scale separation and thus a formulation of critical dynamics in terms of nonlinear Langevin equations: The relevant "slow" modes consist of the order parameter and all conserved quantities to which it is statically or dynamically coupled. All remaining "fast" degrees of freedom are captured through an effective Gaussian white noise. Different values for $z$ ensue depending on whether the order parameter is a conserved quantity or not, and on the additional conserved quantities present. The diffusive relaxation of the latter near criticality can either be characterized by the same exponent $z$ as for the order parameter
PACS numbers: 64.60.Ak, 05.40.-a, 64.60.Ht

("strong" dynamic scaling), or be given by different power laws ("weak" scaling) [5].

In order to ensure relaxation towards thermal equilibrium at long times, as given by a Gibbs distribution, one has to carefully implement detailed-balance conditions. In the language of nonlinear Langevin equations, these are (i) the Einstein relation between the relaxation constants and the noise strengths, and (ii) the condition that the probability current associated with reversible kinetics be divergence free [7]. Naturally, the following question arises: What happens if the equilibrium conditions are violated? In this Letter, we shall explore two generic types of detailed-balance violations, namely (a) coupling the order parameter and additional conserved quantities to heat baths with different temperatures, and (b) allowing for spatially anisotropic noise correlations for conserved variables. To determine their universal features, we map the Langevin dynamics for most of the models listed in Ref. [5] to a dynamic field theory [8], and employ standard renormalization group (RG) methods [6].

In the theory of static critical phenomena, an analogous issue concerns the effect of terms that break the original order parameter symmetry, e.g., the influence of cubic anisotropies on the isotropic $n$-component Heisenberg model. There, the rotational symmetry is restored at criticality, provided $n<n_{c} \approx 4$ [9]. Since detailed balance originates from time-reversal symmetry, we might anticipate that it could, under certain conditions, effectively become reinstated in nonequilibrium critical dynamics, whereupon the asymptotic scaling laws are those of the corresponding equilibrium model. Yet even then, the question arises of whether there exist any nonequilibrium dynamical RG fixed points that could strongly influence crossover regimes. The other possible scenario is of course that violating detailed balance constitutes a relevant perturbation, rendering the equilibrium RG fixed point unstable, and driving the system towards a genuine nonequilibrium stationary state.

The simplest case represents purely relaxational dynam$i c s$, with either nonconserved or conserved, and therefore 
diffusively relaxing, order parameter $\mathbf{S}$ [10] (models $A$ and $B$ according to the classification in Ref. [5], respectively). With the effective LGW Hamiltonian

$$
H[\mathbf{S}]=\int d^{d} x\left[\frac{\tau}{2} \mathbf{S}(x)^{2}+\frac{1}{2}[\nabla \mathbf{S}(x)]^{2}+\frac{u}{4 !} \mathbf{S}(x)^{4}\right],
$$

the corresponding Langevin equations read

$$
\partial_{t} \mathbf{S}(x, t)=-\lambda(i \nabla)^{a} \delta H[\mathbf{S}] / \delta \mathbf{S}(x, t)+\boldsymbol{\zeta}(x, t),
$$

where $a=0$ (2) for models A (B). The noise has zero mean, $\langle\zeta(x, t)\rangle=0$, and its correlations are taken to be

$$
\left\langle\zeta^{\alpha}(x, t) \zeta^{\beta}\left(x^{\prime}, t^{\prime}\right)\right\rangle=2 \tilde{\lambda}(i \nabla)^{a} \delta^{\alpha \beta} \delta\left(x-x^{\prime}\right) \delta\left(t-t^{\prime}\right) .
$$

The equilibrium Einstein relation requires $\tilde{\lambda}=k_{\mathrm{B}} T \lambda$, which ensures that the associated probability distribution for a configuration $\mathbf{S}$ approaches $P_{\mathrm{eq}}[\mathbf{S}] \propto \exp (-H[\mathbf{S}] /$ $\left.k_{\mathrm{B}} T\right)$ as $t \rightarrow \infty$. (Henceforth, we put $k_{\mathrm{B}} T=1$.) Setting $\tilde{\lambda} \neq \lambda$ violates detailed balance; yet this simply represents a temperature shift here. Indeed, upon rescaling $\mathbf{S} \rightarrow(\tilde{\lambda} / \lambda)^{1 / 2} \mathbf{S}$ and $u \rightarrow \tilde{u}=(\tilde{\lambda} / \lambda)^{1 / 2} u$, detailed balance is formally restored, albeit with a different nonlinear static coupling [11]. At criticality, the RG flow will take the latter to the Heisenberg fixed point, independent of its initial value, and thus the equilibrium critical exponents are recovered, namely to lowest nontrivial orders in the deviation $\epsilon=4-d$ from the upper critical dimension $d_{c}=4: \quad \nu^{-1}=2-(n+2) \epsilon /(n+8)$, $\eta=(n+2) \epsilon^{2} / 2(n+8)^{2}[6]$, and $z=2+c \eta$ with $c=6 \ln \frac{4}{3}-1+O(\epsilon)$ for model A, whereas $z=4-\eta$ (exactly) for model B [10]. We already note that the same rescaling with $g^{2} \rightarrow \tilde{g}^{2}=\tilde{\lambda} g^{2} / \lambda$ maps the isotropic $O$ (3)-symmetric nonequilibrium model $\mathbf{J}$ [11]

$$
\begin{gathered}
\partial_{t} \mathbf{S}=-g \delta H[\mathbf{S}] / \delta \mathbf{S} \times \mathbf{S}+\lambda \nabla^{2} \delta H[\mathbf{S}] / \delta \mathbf{S}+\zeta, \\
\left\langle\zeta^{\alpha}(x, t) \zeta^{\beta}\left(x^{\prime}, t^{\prime}\right)\right\rangle=-2 \tilde{\lambda} \nabla^{2} \delta^{\alpha \beta} \delta\left(x-x^{\prime}\right) \delta\left(t-t^{\prime}\right)
\end{gathered}
$$

onto its equilibrium version, with $z=(d+2-\eta) /$ 2 [12].

It is indeed a well-established fact that model A or Glauber dynamics for the kinetic Ising model $(n=1)$ is quite robust against nonequilibrium perturbations $[13,14]$, even when these break the up/down symmetry [15]. Novel features arise only when Kawasaki dynamics is introduced, whereupon the order parameter becomes conserved (model B), and in addition the noise strength is rendered anisotropic: $\tilde{\lambda} \nabla^{2} \rightarrow \tilde{\lambda}_{\|} \nabla_{\|}^{2}+\tilde{\lambda}_{\perp} \nabla_{\perp}^{2}$ in Eq. (3), corresponding to coupling the longitudinal and transverse sectors (of dimensions $d_{\| / \perp}$ ) to heat baths with different temperatures $T_{\| / \perp}$. For this two-temperature or randomly driven model $B$, and for $T_{\|}>T_{\perp}$, only the transverse sector softens at $T_{c}$. As a consequence, the system develops marked anisotropies akin to driven lattice gases (or equilibrium uniaxial dipolar magnets, Lifshitz points, and ferroelastic materials), described by the generalized scaling law $C\left(q_{\|}, q_{\perp}, \omega, \tau\right)=q_{\perp}^{-2-z+\eta} \tilde{C}_{ \pm}\left(q_{\perp} \xi, q_{\|} / q_{\perp}^{1+\Delta}, \omega\right)$ $q_{\perp}^{z}$ ) [1]. As $\Delta \neq 0$, equilibrium isotropic scaling is violated. However, upon omitting irrelevant terms, the Langevin equation at criticality may be written in the form

$$
{ }_{\partial} \mathbf{S}(x, t)=\lambda_{\perp} \nabla_{\perp}^{2} \delta H_{\text {ef } \mathrm{f}}[\mathbf{S}] / \delta \mathbf{S}(x, t)+\boldsymbol{\zeta}(x, t),
$$

with an effective Hamiltonian that contains long-range interactions [16] [here, $\int_{q} \cdots=\int d^{d} q /(2 \pi)^{d} \cdots$ ]

$$
\begin{aligned}
H_{\mathrm{eff}}= & \int_{q} \frac{c q_{\|}^{2}+q_{\perp}^{2}\left(\tau_{\perp}+q_{\perp}^{2}\right)}{2 q_{\perp}^{2}}|\mathbf{S}(q)|^{2} \\
& +\int d^{d} x \frac{\tilde{u}_{\perp}}{4 !} \mathbf{S}^{2} .
\end{aligned}
$$

These strongly affect the nature of the ordered phase, and reduce the upper (to $d_{c}=4-d_{\|}$) as well as lower critical dimension [16,17]. To one-loop order (only), the $\epsilon$ expansion for the static critical exponents formally yields the same results as for the equilibrium Heisenberg model, albeit with $\epsilon=4-d-d_{\|}$. The two-loop results for $d_{\|}=1$ can be found in Ref. [18]. Moreover, the exact scaling relations $\Delta=1-\eta / 2$ and $z=4-\eta$ hold.

We have extended these considerations to models $\mathrm{C}$ and D [19], which take the static coupling

$$
\Delta H[\mathbf{S}, \rho]=\int d^{d} x\left[\frac{1}{2} \rho(x)^{2}+\frac{g}{2} \rho(x) \mathbf{S}(x)^{2}\right]
$$

of the nonconserved/conserved order parameter to the conserved energy density $\rho$ into account [20]. The dynamics is then defined through Eq. (2) with $H[\mathbf{S}, \rho]=H[\mathbf{S}]+$ $\Delta H[\mathbf{S}, \rho]$, setting $a=0(2)$ for model C (D), and

$$
\begin{array}{r}
\partial_{t} \rho(x, t)=D \nabla^{2} \delta H[\mathbf{S}, \rho] / \delta \rho(x, t)+\eta(x, t), \\
\left\langle\eta(x, t) \eta\left(x^{\prime}, t^{\prime}\right)\right\rangle=-2 \tilde{D} \nabla^{2} \delta\left(x-x^{\prime}\right) \delta\left(t-t^{\prime}\right) .
\end{array}
$$

In addition to $\tilde{u}$, there appears now a three-point coupling $f=\tilde{\lambda} g^{2} / \lambda$, also marginal in $d_{c}=4$, as well as the dimensionless ratio of time scales $w=\lambda / D$, and the parameter $\Theta=\tilde{D} \lambda / D \tilde{\lambda}$, which can be interpreted as the temperature ratio $T_{\rho} / T_{S}$ of the heat baths coupled to the energy density and order parameter, respectively. For model $C$, we find the associated RG one-loop beta function $\beta_{\Theta}=-2 \Theta(1-\Theta) f w /(1+w)^{2}$. This result already establishes the stability of the equilibrium fixed point $\Theta_{\mathrm{eq}}^{*}=1$. In fact, we have not found any other, genuinely nonequilibrium RG fixed points corresponding to $\Theta=0$ or $\Theta=\infty$ at all. This remains true even when we allow for spatially anisotropic noise for the conserved field $\rho$, as the RG beta function for the additional new variable $\sigma=\Theta_{\|} / \Theta_{\perp}$ becomes to one-loop order: $\beta_{\sigma}=-\sigma(1-$ $\sigma) f \Theta_{\perp} / 3(1+w)^{3}$, with the isotropic fixed point $\sigma_{\text {is }}^{*}=$ 1 being stable. Thus, there are merely the three (one-loop) equilibrium scaling regimes [20]: (a) Strong-scaling, $n=$ 1: $w^{*}=1, z_{S}=z_{\rho}=2+\alpha / \nu$; (b) weak-scaling, $2 \leq$ $n<4: w^{*}=\infty, z_{S}=2(1+\alpha / n \nu)<z_{\rho}=2+\alpha / \nu$; (c) "model A," $n \geq 4: w^{*}=\infty, z_{s}=2+c \eta, z_{\rho}=2$; here $\alpha=2-d \nu$ denotes the specific-heat critical exponent. In contrast to the above results, a linear coupling of a conserved mode to the order parameter induces effective 
long-range interactions, leading to novel genuine nonequilibrium behavior [21].

For model $D$, the characteristic time scale for the order parameter is always much larger than that of the diffusive field, and therefore $w^{*}=0$. By integrating out the scalar fields $\rho$ from the dynamic functional, one can then readily show that the nonequilibrium parameter $\Theta$ disappears entirely from the theory in the limit $w \rightarrow 0$ [19], which completely reduces the model to the equilibrium theory, with $z_{S}=4-\eta$, whereas $z_{\rho}=2+\alpha / \nu$ for $\alpha>0$ and $z_{\rho}=2$ for $\alpha<0$ ("model B"). Upon allowing for spatially anisotropic order parameter noise correlations, we arrive at a two-temperature model D. As for the corresponding pure order parameter relaxation dynamics, the nonequilibrium perturbations then induce strong anisotropies, whereupon the effective Hamiltonian (7) enters the model-D equations of motion, with only transverse Laplacians, and the associated downwards shift of $d_{c}$. The model-D scaling relations for the anisotropy and dynamic exponents still apply, as well as the two different regimes for $\alpha>0$ and $\alpha<0$, albeit with the static exponents $\nu$, $\eta, \alpha$ of the long-range anisotropic theory [19].

Purely relaxational dynamics provides, however, an insufficient description for many real systems. Often, fully reversible, nondissipative terms originating in the microscopic dynamics need to be taken into account; in isotropic ferromagnets (model $\mathrm{J}$ ), for example, there is also the spin precession in the local magnetic field, see Eq. (4). Such reversible contributions in the Langevin equations for critical dynamics may also involve mode couplings to other conserved and therefore slow variables: In planar ferromagnets, the (nonconserved) order parameter is confined to the $x y$ plane, say, but the nonvanishing commutators of the spin components yield a coupling to the diffusive fluctuations of the conserved $z$ component of the magnetization $\left(\left\langle S_{z}\right\rangle=0\right)$. In isotropic antiferromagnets, the order parameter is represented by the three-component staggered magnetization (not conserved), dynamically coupled to the conserved magnetization. The corresponding Langevin equations define models $E$ and $G$, respectively [22]; their generalization to $n$ order parameter components is termed the Sasvári-Schwabl-Szépfalusy (SSS) model, originally introduced in the context of structural phase transitions $[23,24]$. Last, a consistent description of the critical dynamics near the liquid-gas transition, or equivalently, of the phase separation in binary liquids, involves not only the conserved scalar order parameter density $S$ (a linear combination of the mass and energy densities), but in addition the independent and also conserved transverse corresponding current density $\mathbf{j}_{\perp}$ (model $H$ ) [24,25]. In the following, we describe the effects of nonequilibrium perturbations on these dynamic universality classes with reversible mode couplings $[11,26]$.

The results of our investigations of isotropic and anisotropic nonequilibrium versions of the SSS model were already reported in Ref. [11]; for completeness we review our essential findings here. As in model C, we may choose different effective temperatures for the $n$-component order parameter $\mathbf{S}$ and the $n(n-1) / 2$ noncritical, conserved generators $\mathbf{M}$ of the rotation group $O(n)$. The coupled Langevin equations of motion read

$$
\begin{aligned}
\partial_{t} S^{\alpha}=g \sum_{\beta \neq \alpha} M^{\alpha \beta} S^{\beta}- & \lambda \delta H[S] / \delta S^{\alpha}+\zeta^{\alpha} \\
\left\langle\zeta^{\alpha}(x, t) \zeta^{\beta}\left(x^{\prime}, t^{\prime}\right)\right\rangle=2 \tilde{\lambda} \delta^{\alpha \beta} \delta\left(x-x^{\prime}\right) \delta\left(t-t^{\prime}\right) & \\
\partial_{t} M^{\alpha \beta}= & -g\left(S^{\alpha} \nabla^{2} S^{\beta}-S^{\beta} \nabla^{2} S^{\alpha}\right)+D \nabla^{2} M^{\alpha \beta} \\
& +\eta^{\alpha \beta}, \\
\left\langle\eta^{\alpha \beta}(x, t) \eta^{\gamma \delta}\left(x^{\prime}, t^{\prime}\right)\right\rangle= & -2 \tilde{D} \nabla^{2} \delta\left(x-x^{\prime}\right) \delta\left(t-t^{\prime}\right) \\
& \times\left(\delta^{\alpha \beta} \delta^{\gamma \delta}-\delta^{\alpha \delta} \delta^{\beta \gamma}\right) .
\end{aligned}
$$

As opposed to the purely relaxational models, there exist genuine nonequilibrium fixed points here, corresponding to temperature ratios $\Theta=T_{M} / T_{S}=0$ and $\infty$. In the former case, there is no feedback to the order parameter, and the critical dynamics is essentially model-A like $\left(z_{S}=\right.$ $z_{M}=2$ ), with anomalous noise correlations $\propto q^{d-2}$ for the generators $\mathbf{M}$. For $\Theta_{N}^{*}=\infty$, the order parameter dynamics does not affect the conserved fields, thus $z_{M}=2$, but is itself strongly influenced by their fluctuations $(w=$ $\lambda / D=\infty$ ). We find $z_{S}=d / 2$, as in equilibrium, but a modified static exponent $\nu^{-1}=2-2(n+2) \epsilon /(n+8)$ [11]. However, both these fixed points are unstable, and the asymptotic critical properties are governed by the equilibrium strong-scaling fixed point with $z_{S}=z_{M}=d / 2$ $[23,24]$. Allowing for spatially anisotropic noise for the generators $M$ provides the new parameter $\sigma=\Theta_{\|} / \Theta_{\perp}$, but does not change the overall picture: The isotropic fixed point $\sigma_{\text {is }}^{*}$ remains stable, whence the equilibrium critical behavior is eventually recovered [11]. The anisotropic fixed points are essentially determined by a combination of the $\Theta=0$ and $\Theta=\infty$ characteristics in the different spatial sectors.

The intriguing question now arises how nonequilibrium perturbations affect the critical dynamics of a conserved order parameter field, when reversible mode couplings are present. We have therefore investigated the nonequilibrium model $H$, as defined by [26]

$$
\begin{gathered}
\partial_{t} S=-g(\nabla S) \cdot \mathbf{j}+\lambda \nabla^{2}\left(\tau-\nabla^{2}+\frac{u}{6} S^{2}\right) S+\zeta, \\
\left\langle\zeta(x, t) \zeta\left(x^{\prime}, t^{\prime}\right)\right\rangle=-2 \tilde{\lambda} \nabla^{2} \delta\left(x-x^{\prime}\right) \delta\left(t-t^{\prime}\right), \\
\partial_{t} \mathbf{j}_{\perp}=\mathbf{T}\left[g(\nabla S)\left(\tau-\nabla^{2}\right) S+D \nabla^{2} \mathbf{j}+\eta\right], \\
\left\langle\eta(x, t) \cdot \eta\left(x^{\prime}, t^{\prime}\right)\right\rangle=-2 d \tilde{D} \nabla^{2} \delta\left(x-x^{\prime}\right) \delta\left(t-t^{\prime}\right),
\end{gathered}
$$

where $\mathbf{T}(\vec{q})=\delta_{i j}-q_{i} q_{j} / q^{2}$ denotes the transverse projector in momentum space, and we have omitted irrelevant (in the RG sense) terms. As in model D, asymptotically $w=\lambda / D \rightarrow 0$; as a consequence, only one additional nonequilibrium fixed point $\Theta_{N}^{*}=0$ is 
allowed. In analogy with the corresponding SSS model fixed point, the order parameter dynamics is model-B like, $z_{S}=4$, while $z_{j}=2$, with anomalous noise correlations again $\propto q^{d-2}$ in Fourier space [26]. Yet from $\beta_{\Theta}=-(1-\Theta) \frac{2}{3} \tilde{f}(1+1 / 16 \Theta)$, where $\tilde{f}=\tilde{\lambda} g^{2} / \lambda^{2} D$, we infer that once more the equilibrium weakscaling fixed point $\Theta_{E}^{*}=1$ is stable, with $z_{S}=4-18 \epsilon /$ 19 and $z_{j}=2-\epsilon / 19$ (Galilean invariance fixes $z_{S}+$ $z_{j}=d+2$ ) $[24,25]$. However, the generalization to spatially anisotropic noise, $\tilde{\lambda} \nabla^{2} \rightarrow \tilde{\lambda}_{\|} \nabla_{\|}^{2}+\tilde{\lambda}_{\perp} \nabla_{\perp}^{2}$ and $\tilde{D} \nabla^{2} \rightarrow \widetilde{D}_{\|} \nabla_{\|}^{2}+\widetilde{D}_{\perp} \nabla_{\perp}^{2}$ has a drastic effect. As in the corresponding models $\mathrm{B}$ and $\mathrm{D}$, in this two-temperature model $H$, the characteristic anisotropic DDS singularities emerge. In combination with the mode-coupling terms, this prevents the system from approaching an effective equilibrium model: The divergence-free condition for the reversible probability current cannot be satisfied. In fact, to one-loop order at least we even find a runaway flow for the RG couplings, and are thus unable to determine the long-time scaling behavior [26]. Remarkably, the similarly constructed anisotropic, two-temperature model $J$ is plagued by the same pathology [11], as is a recently studied uniformly rather than randomly driven model $J$ [27]. In that instance, computer simulations revealed that the system displays spatiotemporal chaos at long times; perhaps the absence of an RG fixed point in the two-temperature models $\mathrm{J}$ and $\mathrm{H}$ may indicate chaotic behavior as well.

We have investigated the effect of detailed-balance violations on critical dynamics. Generally, models with nonconserved order parameter are quite robust against nonequilibrium perturbations. The relaxational models A and C only have an equilibrium fixed point. For the SSS model, comprising models $\mathrm{E}$ and $\mathrm{G}$, genuine nonequilibrium fixed points do exist, corresponding to unidirectional couplings between order parameter and conserved fields, but are unstable. Thus, at criticality, the standard critical behavior is eventually recovered. This remains true even when the conserved noise is rendered spatially anisotropic. Essentially the same statements apply for models $\mathrm{B}, \mathrm{D}, \mathrm{J}$, and $\mathrm{H}$ with conserved order parameter, provided detailed-balance violations are introduced isotropically. With spatially anisotropic order parameter noise correlations, however, we find (to one-loop order) no $\mathrm{RG}$ fixed points for models $\mathrm{J}$ and $\mathrm{H}$ with reversible mode-coupling terms. In contrast, the two-temperature relaxational models $\mathrm{B}$ and $\mathrm{D}$ are asymptotically described by an effective equilibrium model, with characteristic anisotropic, long-range correlations.

U.C.T. is grateful to Z. Rácz for introducing him to this field, and acknowledges support from the NSF (DMR0075725) and the Jeffress Memorial Trust (J-594). J.E. S. acknowledges support from the DFG (SFB 413/TP C6), and the EU (ERB-FMBI-CT 97-2816). We thank E. Frey, H. K. Janssen, B. Schmittmann, F. Schwabl, and R. K.P. Zia for illuminating discussions.
[1] B. Schmittmann and R. K. P. Zia, in Phase Transitions and Critical Phenomena, edited by C. Domb and J. L. Lebowitz (Academic Press, London, 1995), Vol. 17.

[2] T. Halpin-Healy and Y.-C. Zhang, Phys. Rep. 254, 215 (1995); J. Krug, Adv. Phys. 46, 139 (1997).

[3] D. S. Fisher, Phys. Rep. 301, 113 (1998).

[4] J. Marro and R. Dickman, Nonequilibrium Phase Transitions in Lattice Models (Cambridge University Press, Cambridge, 1999).

[5] P. C. Hohenberg and B. I. Halperin, Rev. Mod. Phys. 49, 435 (1977).

[6] See, e.g., J. Zinn-Justin, Quantum Field Theory and Critical Phenomena (Clarendon Press, Oxford, 1989).

[7] R. Graham, Springer Tracts in Modern Physics (SpringerVerlag, Berlin, 1973), Vol. 66; U. Deker and F. Haake, Phys. Rev. A 11, 2043 (1975).

[8] C. De Dominicis, J. Phys. (Paris) Colloq. C 1, 247 (1976); H. K. Janssen, Z. Phys. B 23, 377 (1976); R. Bausch, H. K. Janssen, and H. Wagner, ibid. 24, 113 (1976).

[9] I. J. Ketley and D. J. Wallace, J. Phys. A 6, 1667 (1974); E. Brézin, J. C. LeGuillou, and J. Zinn-Justin, Phys. Rev. B 10, 892 (1973); T. Nattermann and S. Trimper, J. Phys. A 8, 2000 (1975).

[10] B. I. Halperin, P. C. Hohenberg, and S.-k. Ma, Phys. Rev. Lett. 29, 1548 (1972); Phys. Rev. B 10, 139 (1974); C. De Dominicis, E. Brézin, and J. Zinn-Justin, ibid. 12, 4945 (1975).

[11] U. C. Täuber and Z. Rácz, Phys. Rev. E 55, 4120 (1997); U. C. Täuber, J. E. Santos, and Z. Rácz, Eur. Phys. J. B 7, 309 (1999); 9, 56 (1999).

[12] S.-k. Ma and G. F. Mazenko, Phys. Rev. Lett. 33, 1383 (1974); Phys. Rev. B 11, 4077 (1975).

[13] F. Haake, M. Lewenstein, and M. Wilkens, Z. Phys. B 55, 211 (1984).

[14] G. Grinstein, C. Jayaprakash, and Y. He, Phys. Rev. Lett. 55, 2527 (1985).

[15] K.E. Bassler and B. Schmittmann, Phys. Rev. Lett. 73, 3343 (1994).

[16] B. Schmittmann and R. K. P. Zia, Phys. Rev. Lett. 66, 357 (1991).

[17] K. E. Bassler and Z. Rácz, Phys. Rev. Lett. 73, 1320 (1994); Phys. Rev. E 52, R9 (1995).

[18] B. Schmittmann, Europhys. Lett. 24, 109 (1993).

[19] V. K. Akkineni and U.C. Täuber (to be published).

[20] E. Brézin and C. De Dominicis, Phys. Rev. B 12, 4954 (1975); B. I. Halperin, P. C. Hohenberg, and S.-k. Ma, ibid. 13, 4119 (1976).

[21] G. Grinstein, C. Jayaprakash, and J.E. S. Socolar, Phys. Rev. E 48, R643 (1993).

[22] B. I. Halperin, P. C. Hohenberg, and E. D. Siggia, Phys. Rev. B 13, 1299 (1976); R. Freedman and G. F. Mazenko, ibid. 13, 4967 (1976).

[23] L. Sasvári, F. Schwabl, and P. Szépfalusy, Physica (Amsterdam) 81A, 108 (1975); H. K. Janssen, Z. Phys. B 26, 187 (1977).

[24] C. De Dominicis and L. Peliti, Phys. Rev. B 18, 353 (1978).

[25] E. D. Siggia, B. I. Halperin, and P. C. Hohenberg, Phys. Rev. B 13, 2110 (1976).

[26] U.C. Täuber and J. E. Santos (to be published).

[27] J. Das, M. Rao, and S. Ramaswamy, e-print cond-mat/ 0104566. 